\title{
Decentralised control of material or traffic flows in networks using phase-synchronisation
}

\author{
Stefan Lämmer ${ }^{a}$, Hiroshi Kori ${ }^{b}$, Karsten Peters ${ }^{a}$, and \\ Dirk Helbing ${ }^{\mathrm{a}, \mathrm{c}}$ \\ a Technische Universität Dresden, \\ Andreas-Schubert-Str. 23, D-01062 Dresden, Germany \\ b Fritz-Haber-Institut der Max-Planck-Gesellschaft, \\ Faradayweg 4-6, D-14195 Berlin, Germany \\ c Collegium Budapest - Institute for Advanced Study, \\ Szentháromság utca 2, H-1014 Budapest, Hungary
}

\begin{abstract}
We present a self-organising, decentralised control method for material flows in networks. The concept applies to networks where time sharing mechanisms between conflicting flows in nodes are required and where a coordination of these local switches on a system-wide level can improve the performance. We show that, under certain assumptions, the control of nodes can be mapped to a network of phase-oscillators.

By synchronising these oscillators, the desired global coordination is achieved. We illustrate the method in the example of traffic signal control for road networks. The proposed concept is flexible, adaptive, robust and decentralised. It can be transferred to other queuing networks such as production systems. Our control approach makes use of simple synchronisation principles found in various biological systems in order to obtain collective behaviour from local interactions.
\end{abstract}

Key words: synchronisation, phase-oscillators, traffic light control, adaptive control

PACS: 89.65.Gh, 45.70.Vn,05.45.Xt

If you want to cite this report, please use the following reference instead:

S. Lämmer, H. Kori, K. Peters, and D. Helbing (2006)

Decentralised control of material or traffic flows in networks

using phase-synchronisation, Physica A 363(1) pp. 39-47 


\section{Introduction}

Efficient control of multiple material flows on a system wide level is one of the most challenging problems in complex socio-technical transportation systems. For the majority of all material flow systems, a parallel service of several intersecting flows or conflicting tasks is impossible, unsafe or inefficient. Alternating exclusion of competing tasks is frequently observed at crossroads in road traffic $[1,2,3,4,5,6,7]$, in the organisation of production processes $[8,9,10,11]$ or in communication networks $[12,13]$. Instead of parallel processing of different flows, a sequential processing must be organised in an optimal manner. Whereas the setup of optimal schedules for single nodes is usually done by traditional optimisation techniques, the control problem on a network-wide level becomes often practically unsolvable by these methods, especially in larger networks. Moreover, most of the material flow networks are subject to continuous demand variations and unforeseen failures. Besides adaptivity and optimality, robustness and flexibility are important requirements for control concepts. Can we learn from the stable, smooth, and efficient flow of nutrients and other chemical substances in circulatory systems of biological organisms? Synchronised dynamics of a population of cells often plays an important role for it $[14,15,16]$. For example, our heart functions as a pump through the appropriate synchronised dynamics of a population of cardiac cells. This synchronisation is realised through appropriate designs of cardiac cells and their network architecture of local interactions. Another interesting example is found in amoeboid organisms $[17,18]$, where the rhythmic contraction pattern produces streaming of protoplasm. Synchronisation phenomena have been intensively studied for these biological systems during the last decades by means of mathematical models, in particular coupled phase-oscillator models $[14,19,20,21,22]$. The great advantage of phase-oscillator models are their tractability and universality.

In the present paper, we take advantage of this tractability and propose a decentralised control principle for material flow networks with transportation delays and setup-times, based on the phase-synchronisation of oscillatory services at the network nodes.

\subsection{Decentralised control using phase-synchronisation}

Let us consider a material transport network, which is a directed graph with of a set of nodes and links. Material can move between the nodes with a finite velocity. Thus, any element experiences a delay $t_{i j}$ between its departure at one node $i$ and its arrival at the next node $j$. Whereas a distinct subset of nodes may act as a source or sink of moving material, we shall concentrate on 
those nodes where the flow of material is conserved, i.e.

$$
\sum q^{\text {in }}=\sum q^{\text {out }}
$$

Here $\sum q^{\text {in }}$ and $\sum q^{\text {out }}$ denote the average rate of incoming and outgoing material, respectively. Each node has to organise the routing of materials arriving through incoming links towards its outgoing links. All allowed connections between incoming and outgoing links can be described through discrete states of the respective node. As long as such a state is 'active', material can flow from a subset of incoming links through the node and leave through outgoing links. All other flow relations are blocked. Usually the switching between different discrete states needs a certain time interval $\tau$, called switching- or setup-time. Depending on the flow rates, the duration of these discrete states may vary. Since we assume a cyclic service sequence, we can assign a periodic motion to every switching node. Thus, a node can be modelled as a hybrid system consisting of a phase-oscillator and a piecewise constant function $M$ that maps the continuous phase-angle $\varphi(t)$ to the discrete service state $s(t)$, e.g. $M: \varphi(t) \rightarrow s(t)$.

The switched service of different flows leads to convoy formation processes. This implies highly correlated arrivals at subsequent nodes, which requires to optimise $M$ with respect to a minimal delay of the material. Whereas the map $M$ can be optimised according to the actual local demand, the phaseangle $\varphi$ is coupled to the oscillatory system of the neighbouring nodes. Thus, with a suitable synchronisation mechanism we can achieve a coordination of the switching states on a network-wide level. In consequence, we suggest an adaptive decentralised control concept consisting of two parts:

(a) Phase-synchronisation of all oscillators in the network, based on local coupling between immediate neighbours.

(b) Mapping of phase-angles to the switching states based on local optimisation.

For the sake of concreteness, we apply our method to the control of traffic lights at intersections of road networks. The rest of this paper is organised as follows: At first, in Sec. 2 we propose a hybrid phase-oscillator model for the intersections of a road network. In Sec. 3 we discuss the synchronisation of the oscillators by a suitable local coupling mechanism for arbitrary network architectures. Finally, in Sec. 4 we show how an optimal switching of green and red traffic light phases with respect to minimum delay times can be reached, based on local adjustment and optimisation. 


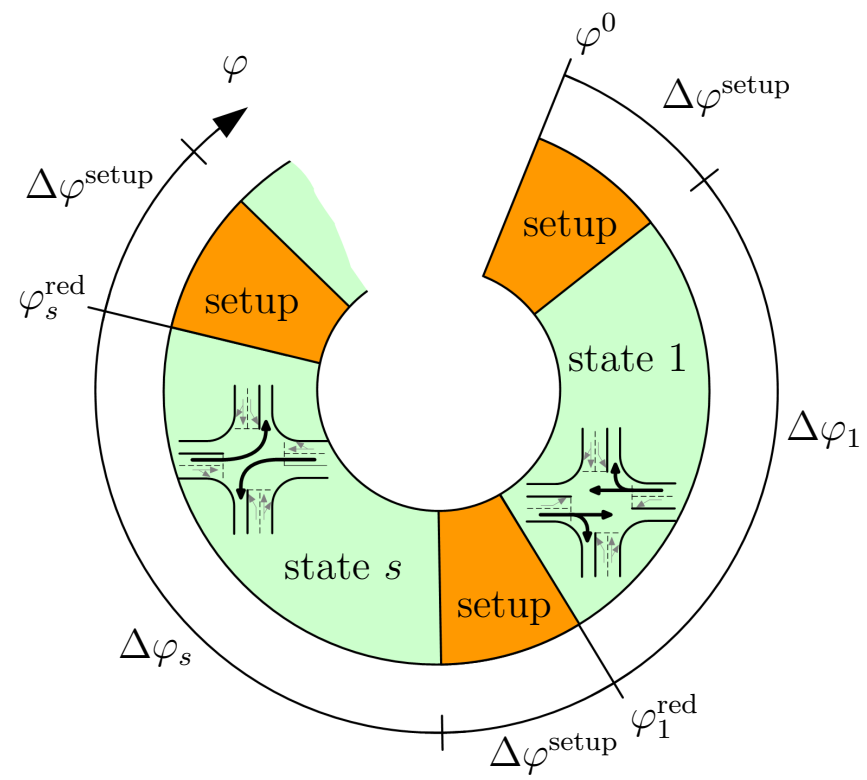

Fig. 1. A single intersection adjusts the mapping of the phase-angle $\varphi$ to the switching states $s$ locally. Within a complete cycle, each state $s$ is sequentially activated for a period $\Delta \varphi_{s}$, during which the corresponding non-conflicting traffic lights are set to green. While switching from one state to another, all traffic lights are set to red for a period of $\Delta \varphi^{\text {setup }}$. The phase-angle, at which a new cycle starts, is denoted by $\varphi^{0}$.

\section{Hybrid model for traffic light control}

In the following, we introduce a hybrid oscillator model for a single traffic light controlled intersection and derive an upper bound for the allowed oscillator frequency.

\subsection{Model of an intersection}

An intersection in road traffic is given by a set of traffic lights $l \in \mathcal{L}$, each one controlling the vehicular flow of a single or several lanes. If two driving paths intersect, we call the related green lights conflicting. We require that conflicting traffic lights are not set to green at the same time. In order to increase the throughput of an intersection, non-conflicting traffic lights can be switched collectively. Such a collective switching of a subset $\mathcal{L}_{s} \subset \mathcal{L}$ of traffic ligths corresponds to one discrete state $s \in\{1,2, \ldots S\}$ of the intersection. An explanatory sketch of this model is shown in Fig. 1.

While the phase-angle $\varphi$ of the oscillator modelling the intersection progresses from 0 to $2 \pi$ at the rate $\omega$, all states $s$ are sequentially activated for some period $\Delta \varphi_{s}$. Thus, all traffic lights in the subset $\mathcal{L}_{s}$ are set to green for this interval 
$\Delta \varphi_{s}$ whereas the remaining lights $l \in \mathcal{L} \backslash \mathcal{L}_{s}$ are turned to red. Moreover, for switching from one state to another, all traffic lights must be set to red for a time-period of $\Delta \varphi^{\text {setup }}(\omega)=\tau \omega$ due to security reasons [1]. These intervals correspond to a switching- or setup-time $\tau$ (see Fig. 1). In conclusion, we obtain

$$
\sum_{s=1}^{S}\left(\Delta \varphi^{\text {setup }}+\Delta \varphi_{s}\right)=2 \pi
$$

\subsection{Maximum oscillator frequency}

To fulfil the balance condition Eq. (1) we have to guarantee that the number of vehicles arriving during a cycle at the intersection is equal to the number of vehicles that can pass the intersection and depart.

The instantaneous vehicular flow $q_{l}(\varphi)$ is given by the number of vehicles $d n_{l}$ that 'potentially' arrive at traffic light $l$ during $(\varphi, \varphi+d \varphi)$, which is equal to the time-shifted flow measured at a cross-section sufficiently far upstream the traffic light.

In the following we do not require any model for the dynamics of $d q_{l}$ thus the control concept is independent of the underlying traffic model. Since the traffic situation usually changes slowly compared to the period of a full switching cycle we assume the average flows $\bar{q}_{l}=1 / 2 \pi \int_{0}^{2 \pi} q_{l}(\varphi) d \varphi$ to be constant. The departure rate during green light is $q_{l}^{\max }$ if there are waiting vehicles, and $q_{l}(\varphi)$ otherwise. If the traffic light shows red, the departure rate is zero. As a consequence, we obtain a lower bound for the green time periods $\Delta \varphi_{s}$.

$$
\Delta \varphi_{s} \geq 2 \pi \max _{l \in \mathcal{L}_{s}} \bar{q}_{l} / q_{l}^{\max }
$$

Here $\bar{q}_{l} / q_{l}^{\max }$ is the utilisation of the lanes, which are served during state $s$. For the signal control to function well, we require $\bar{q}_{l} / q_{l}^{\max }<1$. If $q_{l}=q_{l}^{\max }$ for some $l \in \mathcal{L}_{s}$, we have $\Delta \varphi_{s}=2 \pi$, which implies that state $s$ must be set to green all the time in order to allow for the departure of all vehicles arriving from lane $l$. In this case, our synchronisation strategy would fail to switch to other states, while a purposeful operation of an intersection requires a switching through all $S$ states in one cycle.

The load of the whole intersection is determined by

$$
u=\sum_{s=1}^{S} \max _{l \in \mathcal{L}_{s}} \bar{q}_{l} / q_{l}^{\max } .
$$


Additionally, the conditions imposed by Eq. (3) and Eq. (2) must be satisfied. This is guaranteed only if

$$
\omega \leq \frac{2 \pi}{S \tau}\left(1-\sum_{s=1}^{S} \max _{l \in \mathcal{L}_{s}} \bar{q}_{l} / q_{l}^{\max }\right)=: \quad \omega^{\max }=\frac{2 \pi}{S \tau}(1-u) .
$$

In other words, there exists an upper bound $\omega^{\max }$ for the switching frequency, determined by the amount of time which is spent on setups during a cycle while the balance condition Eq. (1) is fulfilled. For an increasing load u, the overall time for setups must be reduced relative to the duration of a cycle. This suggests to decrease $\omega^{\max }$ for increasing loads $u$. If $u=1$, the maximum frequency becomes $\omega^{\max }=0$. In this case, the intersection is blocked, since there exists no switching cycle which allows all arriving vehicles to depart. This is the typical behaviour of all queuing systems, if the inflow reaches the maximum capacity ${ }^{1}$. Small values of the load $u$ allow for a higher maximum frequency $\omega^{\max }$ and perhaps also a higher cycle frequency $\omega \leq \omega^{\text {max }}$, which is in favour of smaller delays of vehicles. This is obvious, as the maximum delay time of a vehicle is given by the duration of a full cycle.

\section{Global coordination of intersections by synchronisation}

The objective of our decentralised control method is the network wide coordination of the individual switching sequences based on a local coupling between the intersections in the road network. By modelling each intersection $i$ as an oscillator, characterised by its phase-angle $\varphi_{i}$ and its effective frequency $\omega_{i}=\dot{\varphi}$, coordination is achieved by synchronising the oscillator network. Hereby, for providing a common time-scale and allowing the intersections to trigger the switching cycles right at the best time (see Sec. 4), we require a phase-locked state where the phase-difference between neighbouring oscillators is fixed [14]. Therefore, we suggest a coupling between any oscillator $i=1,2, \ldots N$ and its nearest neighbours $j \in \mathcal{N}_{i}$ with adjustments of phases and frequencies on two different timescales.

At first we consider the adaption of the phase-angle $\varphi_{i}$ :

$$
\dot{\varphi}_{i}=\min \left\{\omega_{i}^{\max }, \quad \Omega_{i}(t)+\frac{1}{T_{\varphi}} \sum_{j \in \mathcal{N}_{i}} \sin \left(\varphi_{j}(t)-\varphi_{i}(t)\right)\right\}=: \omega_{i}(t)
$$

where $\Omega_{i}$ is the inherent frequency. As long as $\omega_{i}<\omega_{i}^{\max }, \varphi_{i}$ tries to adjust to the neighbouring phase-angles $\varphi_{j}$. The constant $T_{\varphi}$ corresponds to the typical

$\overline{1}$ In our case, the capacity of an intersection is determined by the maximum departure rates $q_{l}^{\max }$, the switching times $\tau$ and the partition $\left\{\mathcal{L}_{s}\right\}$. 
time-scale for this adaption.

Beyond the phase-synchronising interaction given by $\sin \left(\varphi_{j}(t)-\varphi_{i}(t)\right)$, a second decentralised coupling can be used to increase the inherent frequencies to approach the possible maximum within a slow time-scale:

$$
\dot{\Omega}_{i}=\frac{1}{T_{\Omega}}\left(\min _{j \in \mathcal{N}_{i}}\left\{\omega_{j}(t)\right\}+\Delta \Omega-\Omega_{i}(t)\right) .
$$

Here the constant parameter $\Delta \Omega>0$ provides a linear drift towards higher frequencies.

\subsection{Synchronisation dynamics and network size}

If the coupling mechanisms outlined above are applied to a network of oscillators, two different adaptive behaviours of the system can be distinguished. Either, the system can evolve freely and increase the common frequency, i.e. a slow frequency adaption is possible. Otherwise, the possible dynamics of the coupled system is restricted by an intersection $i_{0}$, whose maximum frequency is reached. This requires the remaining oscillators to be frequency-locked to $\omega_{i_{0}}^{\max }$. Fig. 2 shows an example of both dynamical regimes which are discussed in the following.

(i) Frequency adaption.

As long as $\omega_{i}<\omega_{i}^{\max }$, a synchronised solution is obtained:

$$
\varphi_{i}(t)=\varphi_{j}(t), \quad \omega_{i}(t)=\Omega_{i}(t), \quad \text { and } \quad \dot{\Omega}_{i}=\frac{\Delta \Omega}{T_{\omega}} \quad \forall i
$$

All oscillators have exactly the same phase-angle, while the frequencies $\omega_{i}$ and $\Omega_{i}$ of all oscillators $i$ increase linearly with time. As soon as any oscillator reaches its maximum frequency, however, Eq. (6) is dominated by $\omega_{i}^{\max }$ in the minimum function and $\omega_{i}$ will not increase anymore. Instead, the other state appears.

(ii) Frequency-locked state.

In this state, the effective frequency $\omega_{i_{0}}$ of the oscillator $i_{0}$ with the global minimum of all maximum frequencies is locked to $\omega_{i_{0}}^{\max }=\min _{i^{\prime}} \omega_{i^{\prime}}^{\max }$. In order to achieve synchronisation, the effective frequencies $\omega_{i}$ of all oscillators must become $\omega_{i_{0}}^{\max }$. Under suitable conditions derived below, the frequency-locked state has the solution

$$
\omega_{i}=\omega_{i_{0}}^{\max }=\min _{i^{\prime}} \omega_{i^{\prime}}^{\max } \quad \text { and } \quad \Omega_{i}=\omega_{i_{0}}^{\max }+\Delta \Omega \quad \forall i
$$


The drift parameter $\Delta \Omega$, which was in the previous state (i) responsible for increasing frequencies, must now be compensated by the phase-differences. Using Eqs. (6) and (9) leads to:

$$
\sum_{j \in \mathcal{N}_{i}} \sin \left(\varphi_{j}(t)-\varphi_{i}(t)\right)=-T_{\varphi} \Delta \Omega \quad \forall i \neq i_{0}
$$

Furthermore

$$
\sum_{i=1}^{N} \sum_{j \in \mathcal{N}_{i}} \sin \left(\varphi_{j}(t)-\varphi_{i}(t)\right)=0
$$

which is due to the anti-symmetry of the sin-function. Therefore, the phaseangles of the oscillator $i_{0}$ and its next neighbours $j \in \mathcal{N}_{i_{0}}$ must satisfy

$$
\sum_{j \in \mathcal{N}_{i_{0}}} \sin \left(\varphi_{j}(t)-\varphi_{i_{0}}(t)\right)=(N-1) T_{\varphi} \Delta \Omega
$$

Considering an arbitrary network of $N$ oscillators, there always exists a solution for the immediate neighbours of $i_{0}$ if

$$
(N-1) T_{\varphi} \Delta \Omega \leq 1
$$

A similar condition can be derived for the second-next neighbours and so forth, but for these, the factor in place of $(N-1)$ will become smaller and smaller. Therefore, Eq. (13) gives a sufficient condition for the existence of a phase-locked solution in the frequency-locked state (ii).

Equation (13) exposes an compromise between large network sizes and a fast adapting behaviour. Increasing the network size $N$ requires a smaller adaption rate $\Delta \Omega$ to ensure the synchronisation and vice versa.

From a given initial condition, the system always converges to the phase-locked state (ii), see Fig. 2. It might temporarily enter the frequency adaptation state (i), where the common frequency grows in time to approach the frequency of the slowest oscillator. The transition between states (i) and (ii), which occurs when one of the oscillators reaches its maximum frequency, is smooth. There are no jump-like disturbances in effective frequencies and phase-angles. Once the system converges to state (ii), the synchronised frequency tightly follows variations of the global minimum of $\omega_{i}^{\max }$ frequencies. The phasedifferences between neighbouring oscillators are almost constant. Thus, the phase-synchronisation establishes a load-adaptive common framework among the locally coupled oscillators, which can be used to coordinate the individual switching sequences on a network-wide level. 
(a)

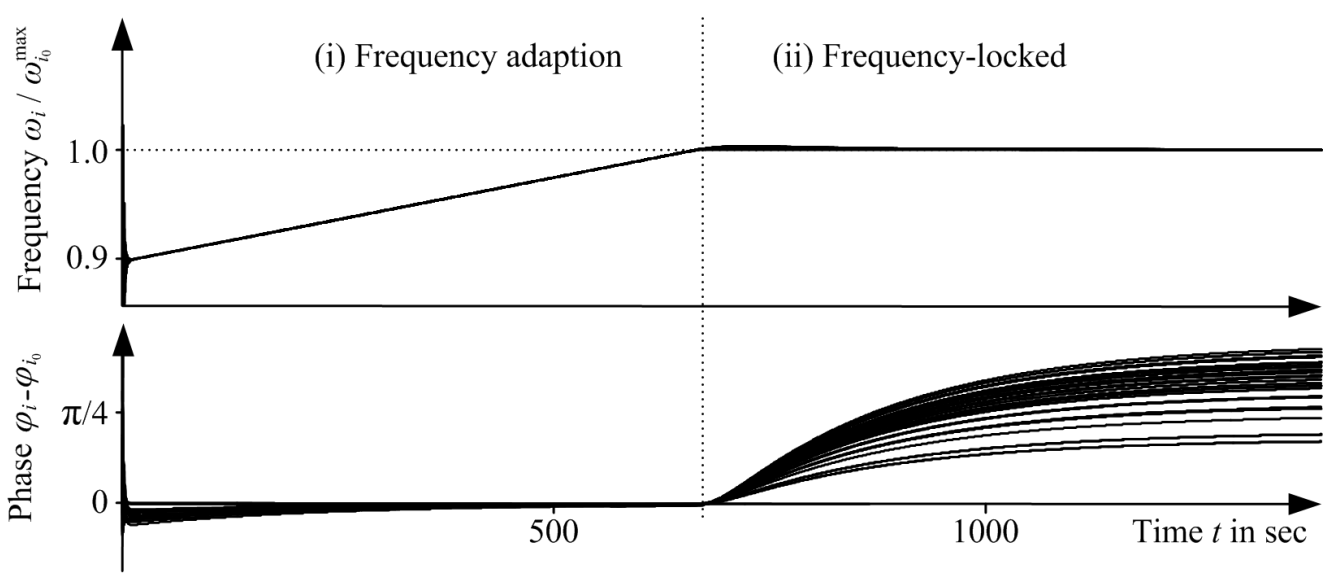

Fig. 2. Simulation results for a regular lattice road network, where the $5 \times 5$ intersections are defined as oscillators with (a) a frequency $\omega_{i}$ and (b) a phase-angle $\varphi_{i}$. Based on a local coupling between immediate neighbours, all oscillators in the network synchronise globally by adjusting their phase-angles mutually. Left: Starting with a random initial condition, the system quickly approaches state (i) with a steadily growing common frequency and vanishing phase-differences. Right: As soon as the maximum possible common frequency is found (indicated by the horizontal dotted line), the system enters state (ii) with a locked common frequency and phase-differences exponentially converging towards constant values. $\left(T_{\varphi}=300 \mathrm{~s}, T_{\Omega}\right.$ $=60 \mathrm{~s}, \omega_{i_{0}}^{\max }=2 \pi / 60 \mathrm{~s}, \Delta \Omega / \omega_{i_{0}}^{\max }=10^{-3}$, and $\left.N=25\right)$

\section{Control of periodic traffic flows at a single intersection}

The time needed by a vehicle to traverse a series of roads controlled by traffic lights is given by the travel times between the intersections and the delay times at red lights. To minimise the overall travel time, or, to increase the throughput of the controlled network, we shall minimise the delay times at every intersection. According to the synchronisation concept outlined in Sec. 3, each intersection $i$ is provided with a phase-angle $\varphi_{i}$, phase-locked to its neighbours and synchronised to a common frequency $\omega$. Due to frequency synchronisation in steady state, the vehicular flows are basically periodic functions of the phase-angle $\varphi_{i}$. Each intersection $i$ may now independently adjust its switching sequence $M: \varphi(t) \rightarrow s(t)$ based on the local arrivals of the vehicles. Thus, for clarity, we neglect the index $i$ in this section and express time $t$ in terms of $\varphi$.

We shall answer the question, where in the cycle the state $s$ should start, to minimise the total time that all vehicles may need to pass the intersection. Therefore, by $\varphi_{s}^{\text {red }}$ we denote the point where the subset $\mathcal{L}_{s}$ of traffic lights switches to red after a green time period $\Delta \varphi_{s}$.

The delay of a single vehicle is given by the period between its arrival $\varphi^{\text {arr }}$ and 
its departure $\varphi^{\text {dep }}$. A vehicle may arrive at the point $\varphi^{\text {arr }}=\varphi_{s}^{\text {red }}+2 \pi p$ with $0<p \leq 1$. Since the vehicles are not rearranged during the queuing process, it is expected to depart at $\varphi^{\text {dep }}=2 \pi+\varphi_{s}^{\text {red }}-(1-p) \Delta \varphi_{s}$. The resulting delay of the particular vehicle is therefore

$$
\varphi^{\mathrm{dep}}-\varphi^{\mathrm{arr}}=\left(2 \pi-\Delta \varphi_{s}\right)(1-p)
$$

Integrating the delays of all vehicular flows $q_{s}(\varphi)=\sum_{l \in \mathcal{L}_{s}} q_{l}(\varphi)$ served in a state $s$ over a complete cycle gives the delays $D_{s}$ imposed by the subset $\mathcal{L}_{s}$ of traffic lights as a function of $\varphi_{s}^{\text {red }}$ and $\Delta \varphi_{s}$ :

$$
D_{s}\left(\varphi_{s}^{\mathrm{red}}, \Delta \varphi_{s}\right)=\int_{0}^{1}\left(2 \pi-\Delta \varphi_{s}\right)(1-p) q_{s}\left(\varphi_{s}^{\mathrm{red}}+2 \pi p\right) 2 \pi d p .
$$

Now we have to minimise the overall delay

$$
D=\sum_{s=1}^{S} D_{s}\left(\varphi_{s}^{\mathrm{red}}, \Delta \varphi_{s}\right)
$$

produced by an intersection during a cycle.

To identify the optimum value of $\varphi_{s}^{\text {red }}$ and $\Delta \varphi_{s}$ for all $\mathcal{L}_{s} \subset \mathcal{L}$ we consider the following two assumptions:

(i) The sequence of states is given, e.g. by the order of their indices $s=1 \ldots S$.

(ii) The periods $\Delta \varphi_{s}$ are fixed and fulfil the conditions Eqs. (2) and (3).

Then there remains only one degree of freedom, which can be expressed by the phase-angle $\varphi^{0}$ that triggers the switching to state $s=1$ (see Fig. 1). Thus, the end-point $\varphi_{s}^{\text {red }}$ of each state $s$ becomes a function of $\varphi^{0}$ :

$$
\varphi_{s}^{\mathrm{red}}\left(\varphi^{0}\right)=\varphi^{0}+\sum_{s^{\prime}=1}^{S}\left(\Delta \varphi^{\mathrm{setup}}+\Delta \varphi_{s^{\prime}}\right)
$$

Inserting Eq. (17) into Eq. (15) and using Eq. (16) provides us with a periodic function $D\left(\varphi^{0}\right)$ for the total delays $D$ produced at the intersection given the start-phase $\varphi^{0}$. With this function, the optimal start-phase $\hat{\varphi}^{0}$ can be found as $D\left(\hat{\varphi}^{0}\right)=\min _{\varphi} D(\varphi)$. The start-phase $\varphi^{0}$ can be shifted to the optimal value $\hat{\varphi}^{0}$ by an offset to the input argument of the map, e.g. $s=M(\varphi(t)-\delta \varphi(t))$.

By adjusting the start-phases, a mutual adaption of the start times of greenphases at neighbouring intersections is reached. This will ensure a minimum delay for all vehicles which have to pass the intersection. Once the optimum $\hat{\varphi}^{0}$ is reached for two intersections, the switching sequence is repeated periodically until the traffic situation changes and a new optimum is obtained by 
the control concept outlined above. Under certain circumstances, it results in emergent green waves.

\section{Conclusions}

In this paper we have developed a method to reach coordination among the traffic lights of an urban street network. This method is based on a combination of a synchronisation resulting from local interactions and a pure local optimisation:

(1) At each intersection, the maximum frequency is determined that allows one to clear the queues on the incoming road sections within one switching cycle. This frequency is given by Eq. (5) in dependence of the respective road utilisation.

(2) The signal controls of all intersections are eventually adjusted to the minimum of all these frequencies, based on a coupling of neighbouring signals. A decentralised method of adjustment is described by Eq. (6).

Thereby, all intersections of the road network continuously equalise the period of their switching cycles. Thus, the switching sequences and the arrivals rates of the vehicles at all intersections become periodic functions.

(3) It is always tried to increase this frequency in order to reduce the waiting times of vehicles in queues. This is reached by introducing a constant drift, see Eq. (7).

(4) Each cycle is subdivided into different green-phases and setup-times as illustrated in Fig. 1. Note that, within one switching cycle, some roads may be served several times.

(5) The optimal green-phases are obtained by minimising the objective function (16). If a periodic sequence of green lights is used, the optimisation problem becomes a minimisation of a continuous function with a single parameter $\varphi^{0}$. In order to minimise the waiting times of vehicles during red light, the model presented in Sec. 4 determines the optimal startphase $\hat{\varphi}^{0}$ of the switching sequence for each intersection.

Under suitable conditions, our concept allows for the emergence of green waves.

Let's consider the example of a single main road with several intersections and different average flow rates in both directions. Then, the vehicles belonging to the highest traffic flow will always have a green light except for the very first traffic light where the vehicles queued are bundled. The green wave will propagate with the largest group of vehicles.

Although our method is quite promising, it has also some limitations: First, the method for determining the maximum frequencies $\omega^{\max }$ needs to be extended for practical application: In real traffic networks, it is possible that 
the traffic arriving at an intersection exceeds capacity. In this case, it is not possible to remove all waiting vehicles within one cycle. Within the framework outlined above, the intersection would be just blocked. However, this situation can be easily avoided by defining a global minimum of the allowed switching frequencies. If the overload situation occurs temporarily, such a minimum frequency will still guarantee a stable functioning of the concept, but it can not avoid queues that persist for more than one switching cycle. Furthermore, to dissolve existing queues quickly and to increase the robustness with respect to stochastic variations in the average flow rates, the maximum frequencies could be assigned a smaller value than determined by Eq. (5).

Second, the frequency of the synchronised system is likely to decrease with growing system size, so that waiting times can become quite long. Therefore, synchronisation cannot be the only goal in signal control. Instead, it would make sense to break up a large synchronised system into smaller synchronised clusters, which may change their shape and size in the course of time. It would also be reasonable to change the order of green-phases sometimes in response to different traffic patterns (e.g. inbound or outbound traffic during the morning and afternoon rush hours, respectively).

Nevertheless, it is useful to have a method for a self-organised coordination of traffic or material flows in a network, based on local interactions. The next step will be to couple this approach with a concrete simulation of the dynamics of material flows and queue formation in the system, as proposed in Ref. [2].

In summary, the optimal switching of traffic lights in non-trivial road networks is one of the most complex material flow control problems. However, the problem in most material flow networks is similar. In production and manufacturing: road sections in traffic correspond to buffers in production, travel and delay times to cycle or production times, junctions to processing units, and different origin-destination flows in road networks to different product flows. Thus, our approach is easily transferable to the control of production systems or other material flow networks. It is particularly suited for systems of moderate size and load, which applies to many manufacturing systems.

\section{Acknowledgements}

We thank the participants of the Thematic Institute on Information and Material Flows in Complex Networks for inspiring discussions, in particular Dieter Armbruster. D.H. and K.P. kindly acknowledge partial financial support from the DFG project He 2789/5-1 and the EU project MMCOMNET. S.L. is grateful for a scholarship by the Studienstiftung des Deutschen Volkes. H.K is grateful for financial support by the Alexander von Humboldt foundation. 


\section{References}

[1] M. Papageorgiou, Concise Encyclopedia of Traffic and Transportation Systems (Pergamon Press, Oxford, 1991).

[2] D. Helbing, S. Lämmer, P. Lebacque, in: C. Deissenberg, R.F. Hartl (Eds.), Optimal Control and Dynamic Games (Springer, Dortrecht, 2005) 239.

[3] C. Diakaki, V. Dinopoulou, K. Aboudolas, M. Papageorgiou, E. Ben-Shabat, E. Seider, A. Leibov, Transport Res. Board 1856 (2003) 202.

[4] C. Gershenson, Complex Systems, 16(1) (2005) 29.

[5] D. Robertson, R.D. Bretherton, IEEE Trans. Veh. Tech. 40(1) (1991) 1.

[6] K. Sekiyama, Y. Ohashi, JACIII 9(2) (2005).

[7] J.J. Henry, J.L. Farges, in: J.P. Perrin (Ed.), Control, Computers, Communications in Transportation ( Pergamon Press, Oxford, 1989).

[8] D. Helbing, in: G. Radons, R. Neugebauer (Eds.), Nonlinear Dynamics of Production Systems (Wiley, New York, 2004).

[9] K. Peters, J. Worbs, U. Parlitz, H.-P. Wiendahl, in: G. Radons, R. Neugebauer (Eds.), Nonlinear Dynamics of Production Systems, (Wiley, New York, 2004).

[10] B. Rem, D. Armbruster, Chaos 13 (2003) 128.

[11] H. Chen, D.D. Yao, Fundamentals of queueing networks (Springer, New York, 2001).

[12] S. Floyd, Comput. Commun. Rev. 21(5), (1991).

[13] S.H. Low, IEEE/ACM Trans.on Netw. 11(4), (2003), 525.

[14] A. Pikovsky, M. Rosenblum, J. Kurths, Synchronization: A universal concept in nonlinear sciences (Cambridge University Press, Cambridge) 2001.

[15] A.T. Winfree, The Geometry of Biological Time (Springer, New York, 1980).

[16] S. Camazine, J.L. Deneubourg, N.R. Franks, J. Sneyd, G. Theraulaz, E. Bonabeau, Self-Organization in Biological Systems, (Princeton University Press, New Jersey, 2003).

[17] T. Nakagaki, H. Yamada, T. Ueda, Biophys. Chem. 84 (2000) 195.

[18] A. Tero, R. Kobayashi, T. Nakagaki, Physica D 205 (2005) 125.

[19] I. Blekhman, Synchronization in science and technology (Asme Press, New York, 1988).

[20] B. Ermentrout, J. Math. Biol. 15 (1991) 339.

[21] Y. Kuramoto, Chemical Oscillations, Waves, and Turbulence (Springer, New York, 1984).

[22] S.H. Strogatz, Nature 410 (2001) 268. 